East Asian Mathematical Journal Vol. 28 (2012), No. 5, pp. 579-585

http://dx.doi.org/10.7858/eamj.2012.043

\title{
NOTE ON UPPER BOUND SIGNED 2-INDEPENDENCE IN DIGRAPHS
}

\author{
Hye KyUng KIM
}

\begin{abstract}
Let $D$ be a finite digraph with the vertex set $V(D)$ and arc set $A(D)$. A two-valued function $f: V(D) \rightarrow\{-1,1\}$ defined on the vertices of a digraph $D$ is called a signed 2-independence function if $f\left(N^{-}[v]\right) \leq 1$ for every $v$ in $D$. The weight of a signed 2-independence function is $f(V(D))=\sum_{v \in V(D)} f(v)$. The maximum weight of a signed 2independence function of $D$ is the signed 2-independence number $\alpha_{s}{ }^{2}(D)$ of $D$. Recently, Volkmann [3] began to investigate this parameter in digraphs and presented some upper bounds on $\alpha_{s}^{2}(D)$ for general digraph $D$. In this paper, we improve upper bounds on $\alpha_{s}^{2}(D)$ given by Volkmann $[3]$.
\end{abstract}

\section{Introduction}

All digraphs considered in this paper are finite, without loops and multiple arcs. For notation and terminology not defined here, we generally follow [1]. For a digraph $D$, we denote the vertex set of $D$ and the arc set of $D$ by $V(D)$ and $A(D)$, respectively. We say that $u$ is an in-neighbor of $v$ and $v$ is an out-neighbor of $u$ if $u v$ is an $\operatorname{arc}$ of $D$. For a vertex $v \in V(D)$, the sets of in-neighbors and outneighbors of $v$ are called the open in-neighborhood and open out-neighborhood of $v$ are denoted by $N_{D}^{-}(v)$ and $N_{D}^{+}(v)$, respectively. The closed in-neighborhood of $v$ is $N_{D}^{-}[v]=N_{D}^{-}(v) \cup\{v\}$. The numbers $d_{D}^{-}(v)=\left|N_{D}^{-}(v)\right|$ and $d_{D}^{+}(v)=$ $\left|N_{D}^{+}(v)\right|$ are the in-degree and out-degree of $v$, respectively. We use $\delta^{-}=\delta^{-}(D)$, $\Delta^{-}=\Delta^{-}(D), \delta^{+}=\delta^{+}(D)$, and $\Delta^{+}=\Delta^{+}(D)$ to denote the minimum indegree, maximum in-degree, minimum out-degree and maximum out-degree of a vertex in $D$, respectively. For $S \subseteq V(D), D[S]$ denotes the subdigraph induced by $S$. If $S \subseteq V(D)$ and $v \in V(D)$, then $E(S, v)$ is the set of arcs from $S$ to $v$. If $S$ and $T$ are two disjoint vertex sets of a digraph $D$, then $E(S, T)$ is the set of arcs from $S$ to $T$.

Received August 17, 2012; Accepted September 26, 2012.

2000 Mathematics Subject Classification. MSC: 05C69.

Key words and phrases. Signed 2-independence function, signed 2-independence number, directed graph.

This work was supported by research grants from the Catholic University of Daegu in 2011. 
For a function $f: V(D) \rightarrow\{-1,1\}$, the weight of $f$ is defined $w(f)=$ $\sum_{v \in V(D)} f(v)$, and for $S \subseteq V(D)$ we define $f(S)=\sum_{v \in S} f(v)$, so $w(f)=$ $f(V(D))$. For a vertex $v \in V(D)$, we denote $f\left(N^{-}[v]\right)$ by $f[v]$ for notational convenience.

The study of signed 2-independence number of undirected graphs was initiated by Zelink [4] and continued in [2] and elsewhere. Recently, Volkmann [3] began to investigate this parameter in digraphs. Formally, a function $f: V(D) \rightarrow\{-1,1\}$ is called a signed 2-independence function (abbreviated by S2IF) if $f[v] \leq 1$ for every vertex $v \in V(D)$. The singed 2-independence number, denoted by $\alpha_{s}^{2}(D)$, of $D$ is the maximum weight of a S2IF on $D$. We call a S2IF of weight $\alpha_{s}^{2}(D)$ a $\alpha_{s}^{2}(D)$-function on $D$. Volkmann [3] presented some upper bounds on $\alpha_{s}^{2}(D)$ for general digraph $D$,

Throughout this paper, if $f$ is a S2IF of $D$, then we let $P$ and $M$ denote the sets of those vertices in $D$ which are assigned under $f$ the value 1 and -1 , respectively and let $p=|P|$ and $m=|M|$. Then $|V(D)|=p+m$ and $\alpha_{s}^{2}(D)=p-m$.

In this paper, we improve upper bounds on $\alpha_{s}^{2}(D)$ given by Volkmann [3].

\section{Main results}

In this section, we study to improve upper bounds on $\alpha_{s}{ }^{2}(D)$ given by Volkmann [3].

Theorem 2.1. Let $D$ be a digraph of order $n$. If $n_{0}$ is the number of vertices of odd in-degree of $V(D)$, Then

$$
\alpha_{s}{ }^{2}(D) \leq \begin{cases}\frac{\left\{\left(\Delta^{+}+1\right)-2\left\lceil\frac{\delta^{-}}{2}\right\rceil\right\} n-2 n_{0}}{\Delta^{+}+1} & \text { if } \delta^{-} \text {is even } \\ \frac{\left(\Delta^{+}+1\right)-2\left\lceil\frac{\delta^{-}}{2}\right\rceil}{\Delta^{+}+1} n & \text { if } \delta^{-} \text {is odd } .\end{cases}
$$

Proof. Let $f$ be a signed 2-independence function on $D$ for which $\alpha_{s}^{2}(D)=$ $f(V(D))$. Put $P_{0}=\left\{v \in P \mid d^{-}(v)\right.$ is odd $\}, P_{e}=P-P_{0}, M_{0}=\{v \in$ $M \mid d^{-}(v)$ is odd $\}, M_{e}=M-M_{0}$, and let $p_{0}=\left|P_{0}\right|, p_{e}=\left|P_{e}\right|, m_{0}=\left|M_{0}\right|$ and $m_{e}=\left|M_{e}\right|$.

By the condition $f[v] \leq 1$ for each $v \in V(D)$, it follows that

$$
\begin{aligned}
& \text { if } v \in P_{0} \text {, then }|E(P, v)| \leq|E(M, v)|-1 \text {, } \\
& \text { if } v \in P_{e} \text {, then }|E(P, v)| \leq|E(M, v)| \text {, } \\
& \text { if } v \in M_{0} \text {, then }|E(P, v)| \leq|E(M, v)|+1, \\
& \text { if } v \in M_{e} \text {, then }|E(P, v)| \leq|E(M, v)|+2 .
\end{aligned}
$$

Moreover,

$$
\delta^{-} \leq d^{-}(v)=|E(P, v)|+|E(M, v)| .
$$


Now, from (1), (2), (3), (4) and (5), we obtain

$$
\begin{gathered}
|E(M, v)| \geq\left\lceil\frac{\delta^{-}+1}{2}\right\rceil \text { for each } v \in P_{0}, \\
|E(M, v)| \geq\left\lceil\frac{\delta^{-}}{2}\right\rceil \text { for each } v \in P_{e}, \\
|E(M, v)| \geq\left\lceil\frac{\delta^{-}-1}{2}\right\rceil \text { for each } v \in M_{0}, \\
\text { and }|E(M, v)| \geq\left\lceil\frac{\delta^{-}-2}{2}\right\rceil \text { for each } v \in M_{e} .
\end{gathered}
$$

Using (6), (7), (8) and (9), we have

$$
\begin{aligned}
|E(M, P)| & =\sum_{v \in P}|E(M, v)|=\sum_{v \in P_{0}}|E(M, v)|+\sum_{v \in P_{e}}|E(M, v)| \\
& \geq p_{0}\left\lceil\frac{\delta^{-}+1}{2}\right\rceil+\left(p-p_{0}\right)\left\lceil\frac{\delta^{-}}{2}\right\rceil \\
& =(n-m)\left\lceil\frac{\delta^{-}}{2}\right\rceil+p_{0}\left(\left\lceil\frac{\delta^{-}+1}{2}\right\rceil-\left\lceil\frac{\delta^{-}}{2}\right\rceil\right)
\end{aligned}
$$

and

$$
\begin{aligned}
|E(D[M])| & =\sum_{v \in M}|E(M, v)|=\sum_{v \in M_{0}}|E(M, v)|+\sum_{v \in M_{e}}|E(M, v)| \\
& \geq m_{0}\left\lceil\frac{\delta^{-}-1}{2}\right\rceil+\left(m-m_{0}\right)\left\lceil\frac{\delta^{-}-2}{2}\right\rceil \\
& =m\left\lceil\frac{\delta^{-}-2}{2}\right\rceil+m_{0}\left(\left\lceil\frac{\delta^{-}-1}{2}\right\rceil-\left\lceil\frac{\delta^{-}-2}{2}\right\rceil\right) .
\end{aligned}
$$

From (11), we get that

$$
\begin{aligned}
|E(M, P)| & =\sum_{v \in M} d^{+}(v)-|E(D[M])| \\
& \leq m \Delta^{+}-m\left\lceil\frac{\delta^{-}-2}{2}\right\rceil-m_{0}\left(\left\lceil\frac{\delta^{-}-1}{2}\right\rceil-\left\lceil\frac{\delta^{-}-2}{2}\right\rceil\right) .
\end{aligned}
$$

Now, we consider two cases.

Case1 : $\delta^{-}=$even.

It is easy to check that $\left\lceil\frac{\delta^{-}+k}{2}\right\rceil-\left\lceil\frac{\delta^{-}+(k-1)}{2}\right\rceil=1(k=1$ or -1$)$ and $\left\lceil\frac{\delta^{-}}{2}\right\rceil-$ $\left\lceil\frac{\delta^{-}-2}{2}\right\rceil=1$. It implies $(n-m)\left\lceil\frac{\delta^{-}}{2}\right\rceil+p_{0} \leq m \Delta^{+}-m\left\lceil\frac{\delta^{-}-2}{2}\right\rceil-m_{0}$ from (10) and (12).

Since $n_{0}=p_{0}+m_{0}$, we have $m \geq \frac{n\left\lceil\frac{\delta^{-}}{2}\right\rceil+n_{0}}{\Delta^{+}+1}$. Thus

$$
\alpha_{s}^{2}(D)=n-2 m \leq n-2 \frac{n\left\lceil\frac{\delta^{-}}{2}\right\rceil+n_{0}}{\Delta^{+}+1}=\frac{\left\{\left(\Delta^{+}+1\right)-2\left\lceil\frac{\delta^{-}}{2}\right\rceil\right\} n-2 n_{0}}{\Delta^{+}+1} .
$$


Case $2: \delta^{-}=$odd

Since $\left\lceil\frac{\delta^{-}+k}{2}\right\rceil-\left\lceil\frac{\delta^{-}+(k-1)}{2}\right\rceil=0(k=1$ or -1$)$ and $\left\lceil\frac{\delta^{-}}{2}\right\rceil-\left\lceil\frac{\delta^{-}-2}{2}\right\rceil=1$, we get $(n-m)\left\lceil\frac{\delta^{-}}{2}\right\rceil \leq m \Delta^{+}-m\left\lceil\frac{\delta^{-}-2}{2}\right\rceil$, from (10)and (12).

Thus $m \geq \frac{n\left\lceil\frac{\delta^{-}}{2}\right\rceil}{\Delta^{+}+1}$ and

$$
\alpha_{s}^{2}(D)=n-2 m \leq n-2 \frac{n\left\lceil\frac{\delta^{-}}{2}\right\rceil}{\Delta^{+}+1}=\frac{\left(\Delta^{+}+1\right)-2\left\lceil\frac{\delta^{-}}{2}\right\rceil}{\Delta^{+}+1} n .
$$

Corollary 2.2. ([3, Theorem 12]) Let $D$ be a digraph of order $n$. Then

$$
\alpha_{s}^{2}(D) \leq \frac{\Delta^{+}+1-2\left\lceil\frac{\delta^{-}}{2}\right\rceil}{\Delta^{+}+1} n .
$$

Theorem 2.3. Let $D$ be a digraph of order $n$. If $n_{0}$ is the number of vertices of odd in-degree of $V(D)$, Then

$$
\alpha_{s}^{2}(D) \leq \begin{cases}\frac{\left(2\left\lfloor\frac{\Delta^{-}}{2}\right\rfloor+1-\delta^{+}\right) n-2 n_{0}}{\delta^{+}+1} & \text { if } \Delta^{-} \text {is even } \\ \frac{2\left\lfloor\frac{\Delta^{-}}{2}\right\rfloor+1-\delta^{+}}{\delta^{+}+1} n & \text { if } \Delta^{-} \text {is odd. }\end{cases}
$$

Proof. Let $f$ be a signed 2-independence function on $D$ for which $\alpha_{s}{ }^{2}(D)=$ $f(V(D))$. Let $P_{0}, M_{0}, P_{e}, M_{e}, p_{0}, p_{e}, m_{0}$ and $m_{e}$ be defined as in the proof of Theorem 1. From (1), (2), (3), (4) in the proof of Theorem 1, and

$$
\Delta^{-} \geq d^{-}(v)=|E(P, v)|+|E(M, v)| .
$$

Using (1), (2), (3), (4) in the proof of Theorem 1, and (13), we have

$$
\begin{aligned}
|E(P, v)| & \leq\left\lfloor\frac{\Delta^{-}-1}{2}\right\rfloor \text { for each } v \in P_{0}, \\
|E(P, v)| & \leq\left\lfloor\frac{\Delta^{-}}{2}\right\rfloor \text { for each } v \in P_{e}, \\
|E(P, v)| & \leq\left\lfloor\frac{\Delta^{-}+1}{2}\right\rfloor \text { for each } v \in M_{0}, \\
\text { and }|E(P, v)| & \leq\left\lfloor\frac{\Delta^{-}+2}{2}\right\rfloor \text { for each } v \in M_{e} .
\end{aligned}
$$

From (14), (15), (16) and (17), we get

$$
\begin{aligned}
|E(P, M)| & =\sum_{v \in M}|E(P, v)|=\sum_{v \in M_{0}}|E(P, v)|+\sum_{v \in M_{e}}|E(P, v)| \\
& \leq m_{0}\left\lfloor\frac{\Delta^{-}+1}{2}\right\rfloor+\left(m-m_{0}\right)\left\lfloor\frac{\Delta^{-}+2}{2}\right\rfloor \\
& =m\left\lfloor\frac{\Delta^{-}+2}{2}\right\rfloor+m_{0}\left(\left\lfloor\frac{\Delta^{-}+1}{2}\right\rfloor-\left\lfloor\frac{\Delta^{-}+2}{2}\right\rfloor\right)
\end{aligned}
$$


and

$$
\begin{aligned}
|E(D[P])| & =\sum_{v \in P}|E(P, v)|=\sum_{v \in P_{0}}|E(P, v)|+\sum_{v \in P_{e}}|E(P, v)| \\
& \leq p_{0}\left\lfloor\frac{\Delta^{-}-1}{2}\right\rfloor+p_{e}\left\lfloor\frac{\Delta^{-}}{2}\right\rfloor=p_{0}\left\lfloor\frac{\Delta^{-}-1}{2}\right\rfloor+\left(p-p_{0}\right)\left\lfloor\frac{\Delta^{-}}{2}\right\rfloor \\
& =p\left\lfloor\frac{\Delta^{-}}{2}\right\rfloor+p_{0}\left(\left\lfloor\frac{\Delta^{-}-1}{2}\right\rfloor-\left\lfloor\frac{\Delta^{-}}{2}\right\rfloor\right) .
\end{aligned}
$$

From (19), we have

$$
\begin{aligned}
|E(P, M)| & =\sum_{v \in P} d^{+}(v)-|E(D[P])| \\
& \geq p \delta^{+}-p\left\lfloor\frac{\Delta^{-}}{2}\right\rfloor-p_{0}\left(\left\lfloor\frac{\Delta^{-}-1}{2}\right\rfloor-\left\lfloor\frac{\Delta^{-}}{2}\right\rfloor\right) .
\end{aligned}
$$

Now, we consider two cases. Case1 : $\Delta^{-}$is even.

Using (18) and (20),

$$
p \delta^{+}-p\left\lfloor\frac{\Delta^{-}}{2}\right\rfloor+p_{0} \leq m\left\lfloor\frac{\Delta^{-}+2}{2}\right\rfloor-m_{0} .
$$

Substitute $p=n-m$ into $(21),(n-m)\left(\delta^{+}-\left\lfloor\frac{\Delta^{-}}{2}\right\rfloor\right)+p_{0}+m_{0} \leq m\left\lfloor\frac{\Delta^{-}+2}{2}\right\rfloor$.

$$
\begin{gathered}
m\left(\delta^{+}+1\right) \geq n\left(\delta^{+}-\left\lfloor\frac{\Delta^{-}}{2}\right\rfloor\right)+n_{0}, \text { and } m \geq \frac{n\left(\delta^{+}-\left\lfloor\frac{\Delta^{-}}{2}\right\rfloor\right)+n_{0}}{\delta^{+}+1} . \text { It follows that } \\
\alpha_{s}^{2}(D)=n-2 m \leq n-\frac{2\left\{n\left(\delta^{+}-\left\lfloor\frac{\Delta^{-}}{2}\right\rfloor\right)+n_{0}\right\}}{\delta^{+}+1} \\
=\frac{\left(2\left\lfloor\frac{\Delta^{-}}{2}\right\rfloor+1-\delta^{+}\right) n-2 n_{0}}{\delta^{+}+1} .
\end{gathered}
$$

Case $2: \Delta^{-}$is odd.

Using (18) and (20),

$$
p \delta^{+}-p\left\lfloor\frac{\Delta^{-}}{2}\right\rfloor \leq m\left\lfloor\frac{\Delta^{-}+2}{2}\right\rfloor .
$$

Substitute $p=n-m$ into $(22),(n-m)\left(\delta^{+}-\left\lfloor\frac{\Delta^{-}}{2}\right\rfloor\right) \leq m\left\lfloor\frac{\Delta^{-}+2}{2}\right\rfloor$.

Therefore, $n\left(\delta^{+}-\left\lfloor\frac{\Delta^{-}}{2}\right\rfloor\right) \leq m\left(\delta^{+}+1\right)$.

It follows that

$$
\alpha_{s}^{2}(D)=n-2 m \leq n-2 \frac{n\left(\delta^{+}-\left\lfloor\frac{\Delta^{-}}{2}\right\rfloor\right)}{\delta^{+}+1}=\frac{2\left\lfloor\frac{\Delta^{-}}{2}\right\rfloor+1-\delta^{+}}{\delta^{+}+1} n .
$$

Corollary 2.4. ([3, Theorem 13]) If $D$ is a digraph of order $n$, then

$$
\alpha_{s}^{2}(D) \leq \frac{2\left\lfloor\frac{\Delta^{-}}{2}\right\rfloor+1-\delta^{+}}{\delta^{+}+1} n .
$$


Theorem 2.5. Let $D$ be a digraph of order $n$ such that $\delta^{+}-\left\lfloor\frac{\Delta^{-}}{2}\right\rfloor \geq 0$. Let $n_{0}$ be the number of vertices whose in-degree of $V(D)$ is odd and $m_{0}$ the number of vertices whose in-degree is odd and assigned value is -1 . Then

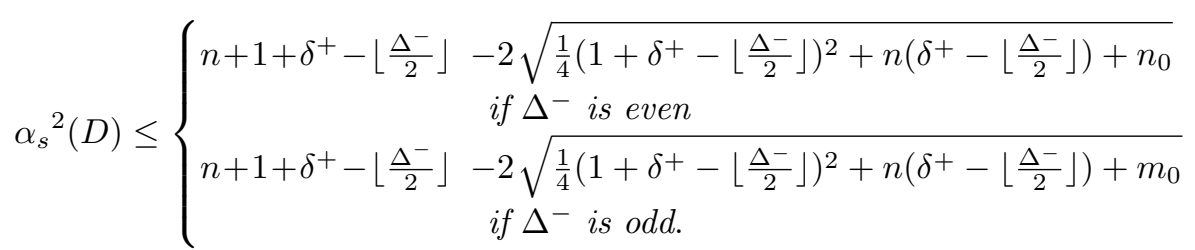

Proof. Let $f$ be a signed 2-independence function on $D$ for which $\alpha_{s}^{2}(D)=$ $f(V(D))$, and let $P_{0}, M_{0}, P_{e}, M_{e}, p_{0}, p_{e}, m_{0}$ and $m_{e}$ be defined as in the proof of Theorem 1. By the definition of S2IF, each vertex of $M_{0}$ has at most $m$ in-neighbors in $P$ and each vertex of $M_{e}$ has at most $(m+1)$ in-neighbors in $P$. Thus $\left|E\left(P, M_{0}\right)\right| \leq m_{0} m$ and $\left|E\left(P, M_{e}\right)\right| \leq\left(m-m_{0}\right)(m+1)$. It follows that

$$
|E(P, M)|=\left|E\left(P, M_{0}\right)\right|+\left|E\left(P, M_{e}\right)\right| \leq m(m+1)-m_{0} .
$$

Using (20) in the proof of Theorem 3,

$$
(n-m)\left(\delta^{+}-\left\lfloor\frac{\Delta^{-}}{2}\right\rfloor\right)+p_{0}\left(\left\lfloor\frac{\Delta^{-}}{2}\right\rfloor-\left\lfloor\frac{\Delta^{-}-1}{2}\right\rfloor\right) \leq m^{2}+m-m_{0} .
$$

Now, we consider two cases.

Case1 : $\Delta^{-}$is even.

$$
(n-m)\left(\delta^{+}-\left\lfloor\frac{\Delta^{-}}{2}\right\rfloor\right)+p_{0} \leq m^{2}+m-m_{0}
$$

Thus

$$
m^{2}+\left(1+\delta^{+}-\left\lfloor\frac{\Delta^{-}}{2}\right\rfloor\right) m-n\left(\delta^{+}-\left\lfloor\frac{\Delta^{-}}{2}\right\rfloor\right)-n_{0} \geq 0
$$

and

$$
m \geq-\frac{1+\delta^{+}-\left\lfloor\frac{\Delta^{-}}{2}\right\rfloor}{2}+\sqrt{\frac{1}{4}\left(1+\delta^{+}-\left\lfloor\frac{\Delta^{-}}{2}\right\rfloor\right)^{2}+n\left(\delta^{+}-\left\lfloor\frac{\Delta^{-}}{2}\right\rfloor\right)+n_{0} .}
$$

Now, we get the bound as follows

$$
\begin{aligned}
\alpha_{s}^{2}(D) & =n-2 m \\
& \leq n+1+\delta^{+}-\left\lfloor\frac{\Delta^{-}}{2}\right\rfloor-2 \sqrt{\frac{1}{4}\left(1+\delta^{+}-\left\lfloor\frac{\Delta^{-}}{2}\right\rfloor\right)^{2}+n\left(\delta^{+}-\left\lfloor\frac{\Delta^{-}}{2}\right\rfloor\right)+n_{0} .}
\end{aligned}
$$

Case $2: \Delta^{-}$is odd. 
Since $(n-m)\left(\delta^{+}-\left\lfloor\frac{\Delta^{-}}{2}\right\rfloor\right) \leq m^{2}+m-m_{0}$, it implies that

$$
m \geq-\frac{1+\delta^{+}-\left\lfloor\frac{\Delta^{-}}{2}\right\rfloor}{2}+\sqrt{\frac{1}{4}\left(1+\delta^{+}-\left\lfloor\frac{\Delta^{-}}{2}\right\rfloor\right)^{2}+n\left(\delta^{+}-\left\lfloor\frac{\Delta^{-}}{2}\right\rfloor\right)+m_{0} .}
$$

So, we get the bound as follows

$$
\begin{aligned}
\alpha_{s}^{2}(D) & =n-2 m \\
& \leq n+1+\delta^{+}-\left\lfloor\frac{\Delta^{-}}{2}\right\rfloor-2 \sqrt{\frac{1}{4}\left(1+\delta^{+}-\left\lfloor\frac{\Delta^{-}}{2}\right\rfloor\right)^{2}+n\left(\delta^{+}-\left\lfloor\frac{\Delta^{-}}{2}\right\rfloor\right)+m_{0} .}
\end{aligned}
$$

Corollary 2.6. ([3, Theorem 14]) Let $D$ be a digraph of order $n$ such that $\delta^{+}-\left\lfloor\frac{\Delta^{-}}{2}\right\rfloor \geq 0$. Then

$$
\alpha_{s}^{2}(D) \leq \begin{cases}n+1+\delta^{+}-\left\lfloor\frac{\Delta^{-}}{2}\right\rfloor & -2 \sqrt{\frac{1}{4}\left(1+\delta^{+}-\left\lfloor\frac{\Delta^{-}}{2}\right\rfloor\right)^{2}+n\left(\delta^{+}-\left\lfloor\frac{\Delta^{-}}{2}\right\rfloor\right)} \\ & \text { if } \Delta^{-} \text {is even } \\ n+1+\delta^{+}-\left\lfloor\frac{\Delta^{-}}{2}\right\rfloor & -2 \sqrt{\frac{1}{4}\left(1+\delta^{+}-\left\lfloor\frac{\Delta^{-}}{2}\right\rfloor\right)^{2}+n\left(\delta^{+}-\left\lfloor\frac{\Delta^{-}}{2}\right\rfloor\right)} \\ & \text { if } \Delta^{-} \text {is odd. }\end{cases}
$$

\section{References}

[1] G. Chartrand and L. Lesniak, Graphs and digraphs, 4th ed. Chapman and Hall, Boca Raton, 2005.

[2] M. A. Henning, Signed 2-independence in graphs, Discrete Math. 250 (2002), 93-107.

[3] L. Volkmann, Signed 2-independence in digraphs, Discrete. Math. 312 (2012), 465-471.

[4] B. Zelinka, On signed 2-independence numbers of graphs, Manuscript.

Hye Kyung Kim

Department of Mathematics Education, Catholic University of Daegu, Kyeongsan 712-702, Republic of Korea

E-mail address: hkkim@cu.ac.kr 